Rev. Psicol. (Arequipa. Univ. Catól. San Pablo) / Año 2021 / Vol 11 / N 1 / pp. 101-126 ISSN 2306-0565 versión impresa / ISSN 2311-7397 versión on line

\title{
Presencia de berrinches en niños y niñas de 2 a 3 años
}

\author{
Presence of Tantrums in Boys and Girls from 2 to 3 Years of Age \\ Fabiola Stefany Tito Abado \\ Institución Educativa Víctor García Hoz, Arequipa Perú \\ (D) https://orcid.org/oooo-0oo1-7523-124X \\ Paola Arlette Cáceres Gallegos \\ Institución Educativa Víctor García Hoz, Arequipa Perú \\ (iD https://orcid.org/oooo-00o1-7518-8878 \\ Charles Portilla Revollar \\ Universidad Católica de Santa María, Arequipa, Perú \\ (iD https://orcid.org/oooo-0oo3-2866-0695 \\ Correspondencia: charlesprı@hotmail.com
}

\section{Resumen}

Se investigó la incidencia y características de berrinches en niños y niñas de 2 y 3 años pertenecientes a los 8 distritos más populosos de Arequipa, Perú. Participaron 284 niños y 271 niñas $(N=555)$, 183 de 2 años ( 96 niños y 87 niñas); 372 de 3 años (188 niños y 184 niñas). El diseño fue descriptivo correlacional. Por medio de las instituciones educativas fueron enviadas 1,325 "encuestas" a las familias; el índice de retorno fue del $42 \%$ (555). Se aplicó una Ficha de Reporte de Conductas (que incluía datos demográficos) donde se identificaban y describían los berrinches preparada por los investigadores. Se encontraron 431 casos con berrinches y 124 casos sin berrinches, la incidencia es alta considerándose que es una muestra comunitaria. No se encontraron diferencias estadísticamente significativas de berrinches y género; sí se halló significativamente mayor intensidad de berrinches en los niños; los berrinches se caracterizaron por presencia de llanto, gritos, arrojar juguetes y tirarse al suelo. Las causas básicas fueron negación de petición de objetos y realización de acciones deseadas. La estrategia más común para detenerlos fueron tratar de calmarlos y cargarlos en brazos. Palabras clave: Berrinches, infancia, niños, niñas. 


\begin{abstract}
Was investigated the incidence and characteristics of tantrums in boys and girls between 2 and 3 years, belonging to 8 districts more populous of Arequipa, Peru. Participated 248 boys and 271 girls $(N=555,183$ were 2 years ( 96 boys and 87 girls) and 372 were 3 years old (188 boys and 184 girls). De design was descriptive correlational, 1325 surveys were sent to the families through schools, the returns percentage was $42 \%$ (555). Was applied a Report Card to describe demographic characteristics, identify and describe characteristics of tantrums prepared by the researchers. Were found 431 cases with tantrums and 124 cases without tantrums, the incidence is high because is a community sample. It was not found significant differences on incidence tantrums between boys and girls; however there is a statistically significant difference between intensity of tantrum, with children presenting the highest intensity of tantrums, unlike girls. The characteristics of tantrums were crying, screams, throw toys and fall to the ground. The basic causes were request of objects and activities denied. The used strategies were calm down and carry him in arms.
\end{abstract}

Keywords: Tantrums, childhood, boys, girls.

\section{Introducción}

El desarrollo socioemocional temprano es una habilidad que emerge formando cercanas y seguras relaciones con padres y compañeros, cuyas experiencias regulan paulatinamente la expresividad de las emociones de formas que son aceptadas social y culturalmente. Parte fundamental de la crianza es el control de las emociones, y su desarrollo está influenciado por factores biológicos y experiencias personales (Darling-Churchil \& Lippman, 2016; Papalia et al., 2001). Desde el nacimiento hasta los 5 años, los niños sientan las bases del desarrollo socioemocional que les permiten expresar sus emociones de manera apropiada hasta que llegan a ser independientes y confortables para explorar sus alrededores (Askeland, 2019. De acuerdo con Ashdown y Bernard (2011) y Thompson (2018), en los tres primeros años de vida los niños alcanzan logros fundamentales en el desarrollo socioemocional. Ellos logran un apego seguro con sus cuidadores, estableciendo las bases de la certeza y seguridad de ser protegidos, alimentados y apoyados emocionalmente, lo cual favorece el desarrollo de la competencia social con sus pares y otros adultos. Los niños empiezan a experimentar un amplio rango de emociones junto a la comprensión cada vez más amplia de los sentimientos e intenciones de los otros, los infantes también hace significativos progresos en la conciencia social y autorregulación de sus emociones e impulsos, aunque todavía les falta un largo camino.

El desarrollo emocional se inicia mediante expresiones básicas como agrado y desagrado, incluso en las primeras investigaciones se refería que los infantes nacían con sólo una emoción vivenciada llamada angustia (Tortosa \& Mayor, 1992). Sin embargo, ahora se conoce que al juzgar 
sus expresiones faciales se pueden identificar distintos tipos de emociones como la alegría, tristeza, interés, miedo e ira. Dentro de las emociones principales se acepta que esta última es una de ellas y se presenta generalmente cuando los infantes se sienten obstaculizados en la satisfacción de sus deseos, los problemas sociales y emocionales tales como frustración, ansiedad y depresión; generalmente relacionados con el rendimiento académico y la aceptación social (Bee, 1992; Rice, 1997; Romano et al., 2010).

Los niños y niñas desde que nacen presentan diferencias en sus reacciones, si bien la explicación parece estar en la forma en que las madres o adultos tratan a los infantes, hay respuestas emocionales que siguen con frecuencia patrones que persisten a través de los años, lo que indica que el temperamento básico es innato (California Childcare Health Program, 2006). En la investigación conocida como el estudio longitudinal de Nueva York (ELNY) Thomas et al., 1970) distinguieron tres patrones temperamentales básicos: niños fáciles, niños difíciles, y niños poco afectuosos.

\section{Los Berrinches}

La vida siempre está acompañada de momentos de ira, ésta es una motivación poderosa y ocasiona molestia, decepción y frustración que es expresada de distintas formas de acuerdo con su edad y temperamento, siendo una de estas expresiones el berrinche (Daniels et al., 2012; Eastman \& Rozen, 2001). En estas ocasiones se descarga el sistema de energía acumulada ante situaciones no aceptadas por el niño, generando que el cuerpo como el estado mental se agiten y tomen la forma de ansiedad y hostilidad, buscando la satisfacción de necesidades frustradas. Eastman y Rozen (2001), y más recientemente Solter (2020), refieren que los berrinches o rabietas son expresiones de ira y enojo propios de la inmadurez del desarrollo emocional, considerándose así, como una forma normal de expresar sentimientos negativos. Por tanto, los berrinches como expresión de ira o enojo pueden ser considerados como formas normales de manifestar sentimientos dolorosos o negativos; pero cuando estas manifestaciones son muy frecuentes e intensas podemos necesitar ayuda especializada para manejarlas. Green et al. (2011) encontraron que no sólo la emoción de la ira se asocia al berrinche, sino también la tristeza, y que los berrinches son una ventana para conocer cómo se expresan y regulan las emociones fuertes. Estos investigadores encontraron que las rabietas son difíciles de manejar una vez que se presentan por la intensa carga emocional que representan y que cuando son excesivos y persisten en el tiempo, pueden predecir futuros desajustes y aún desordenes psicológicos. Dado que son manifestaciones que nos permiten conocer algunas características de las expresiones emocionales, constituyen un fenómeno que debe ser estudiado por la ciencia. Para Davison (2003) y Osterman y Bjorkqvist (2010), los berrinches son definidos por sus manifestaciones conductuales físicas (tirarse al suelo, golpear) y manifestaciones conductuales vocálicas (llorar y 
gritar), donde las agresiones verbales no son usualmente sofisticadas.

El estudio de los berrinches de forma sistematizada fue realizado desde hace largo tiempo, pues ya Goodenough en 1931 (citado por Green et al., 2011) escribió una monografía sobre el enojo en la niñez donde describe las características de los berrinches entre los seis meses y ocho años. Kanner (1935) por su parte, reporta que los berrinches son uno de los malos hábitos más frecuentes y representan el $16 \%$ de los requerimientos en la consulta. De igual manera Geleerd (1944) presentó una investigación afirmando que no había buenas definiciones sobre los berrinches y recomienda que la mejor manera de manejarlos era dejar al niño solo hasta que se calme, pero si el niño tiene tendencia a destruir cosas es mejor que sea removido del lugar, aunque en general afirma que los berrinches no deben ser manejados con firmeza.

La American Academy of Pediatrics (1989) refiere que frecuentemente los niños y niñas tienen berrinches cuando se sienten frustrados consigo mismos. Las frustraciones pueden provenir de diferentes situaciones o eventos, y generalmente los padres y madres no entienden lo que desea. Las explosiones de ira se presentan en todas las edades, en niños y adultos, sólo quea medida que maduramos, vamos aprendiendo a canalizar esa ira con expresiones más acordes con las expectativas del entorno social. Alrededor de los dos años se descubre una manera de descargar el sistema de energía acumulada ante situaciones no aceptadas por el niño, y su cuerpo y su estado mental se agitan tomando la forma de ansiedad y hostilidad, buscando la satisfacción de necesidades frustradas. Si bien se acepta que los berrinches son bastante comunes en los primeros años, plantean un reto para los padres, madres, profesores y personas de su entorno.

Osterman y Bjorkqvist (2004) encontraron en Finlandia, que en el $87 \%$ de los hijos cuyos de padres y madres fueron entrevistados, habían tenido berrinches en algún momento y que el tiempo de duración más frecuente fue entre los $5 \mathrm{y}$ 10 minutos; pero que berrinches de más de media hora se presentaron en solo el $6 \%$ de los casos. Además, el lugar más frecuente donde se presentaban era en el hogar a la hora de vestirlos y/o alimentarlos, el segundo lugar más común fue en los vehículos cuando los transportaban a algún sitio. Parece ser que los berrinches en la niñez se presentan en diversas culturas, por ejemplo, en el pueblo amazónico peruano de los Matsigenkas se describe la presencia de berrinches en los niños (Johnson, 2003, citado por Osterman \& Bjorkqvist, 2004). Igualmente, en descripciones del folklore antiguo de Japón se relatan berrinches en la niñez (Tomm et al., 1990, citados por Osterman \& Bjorkqvist, 2004). Aún más, de Waal (200o, citado por Osterman y Bjorkqvist, 2004) refiere que conductas similares a los berrinches han sido observados en chimpancés.

\section{Características de los Berrinches}

La frustración, decepción o enojo se suelen expresar mediante el llanto, 
gritos, pataletas y hasta agresiones, sobre todo entre los 18 meses hasta los tres años (American Academy of Pediatrics, 1989), todo lo cual aparenta una conducta exagerada, fuera de proporción, aunque comunes en la niñez (Mireault \& Trathan (2007). En el pasado, Kanner (1935) refería que los berrinches se caracterizan principalmente por inicio rápido o gradual de llanto a gritos y zapateo, expresiones de insultos en voz alta, patear el suelo, agitar los brazos, empujar o golpear a las personas del entorno próximo, arrojar objetos, tirarse al suelo y golpearse a sí mismo la cabeza contra las paredes u otro objeto.

Los berrinches pueden considerarse un problema cuando son demasiado frecuentes (varias veces a la semana), son demasiado intensos (llanto exagerado, resistencia a calmarse), están cargados de alta agresividad, duran demasiado tiempo (más de 5 minutos); provoca agresiones, perjudican las relaciones familiares, escapan del control de los cuidadores y hacen daño a otras personas (Eastman \& Rozen, 2001).

Potegal y Davidson (2003) indican que los berrinches oscilan entre los más frecuentes, cuando solo hay llanto exagerado y los menos frecuentes, que llaman la atención, cuando el llanto es acompañado de retención de la respiración, golpearse la cabeza, enrojecimiento de mejillas, dilatación de los ojos y hasta vómitos. Estas reacciones desafortunadamente son frecuentes en la niñez y son una de las quejas más comunes de los padres y madres; por lo tanto, los berrinches intensos pueden ser clasificados dentro de los desórdenes de conducta externalizados. Según Potegal y Davidson (1998) las divergencias sobre si los berrinches son normales en la niñez o si son un aviso de posibles problemas posteriores, implican la identificación algunos factores psicosociales asociados a berrinches extremos tales como enfermedades menores, castigo corporal, depresión materna y clase social pobre.

Potegal etal. (2003) aseveran que la frecuencia de los berrinches es de uno por día y se presentan sobre todo cuando los niños y niñas están cansados, hambrientos, perturbados o indispuestos; aunque inclusive padres y madres experimentados no siempre pueden prevenir berrinches ocasionales. Además, la frecuencia e intensidad de los berrinches varían de acuerdo con el ambiente donde esté el niño, aunque la mayoría de los berrinches se presentan en el hogar. Ellos encontraron que en los leves la duración más común de los berrinches fue de 0.5 a 1 minuto, sobre todo cuando en los 30 segundos iniciales zapatea y se tira al suelo; pero en el $75 \%$ de los berrinches intensos la duración fue entre 1.5 a 5 minutos; considerando queel númeroy variedad de conductas del berrinche aumenta si el berrinche tiene más duración. Si el berrinche persiste más allá de los 3 a 4 minutos, la mayoría de las conductas de berrinche son repeticiones o continuaciones de las conductas ya presentadas. Estos investigadores no encontraron relación entre la intensidad de los berrinches y la ansiedad o pánico de los padres.

Belden et al. (2008) afirman que el 70 $\%$ de los niños entre la edad de 18 y 60 meses presentan berrinches, y que 
las características de los berrinches varían de acuerdo con la relación con depresión, problemas de conducta o conducta saludable. Los investigadores refieren que en los niños preescolares que tengan un promedio de 10 a 20 berrinches en un periodo de 30 días, pueden ser indicadores de tener un problema clínico serio; igualmente si no es capaz de calmarse por sí mismo cuando los episodios son más allá de los 25 minutos. Además, si están asociados a manifestaciones depresivas y otros problemas de conducta.

Novotney (2012) encontró que los berrinches sólo se presentan en menos del 10 $\%$ de la población en edad preescolar, sin importar el género, nivel socioeconómico o etnia y que su presencia podría ser signo alarmante de significativos problemas de conducta que deberían ser afrontados antes que aumenten. De manera semejante, Wakschlag et al. (2012) han encontrado que el $87 \%$ de los preescolares presentan ocasionalmente berrinches, pero los berrinches diarios no son tan típicos y sólo los presentan el $10 \%$ de los preescolares evaluados.

Carlson et al. (2016), al estudiar la irritabilidad y los berrinches en muestras clínicas y de la comunidad, hallaron que en la muestra clínica el $45.9 \%$ tenían berrinches severos, pero sólo $23.8 \%$ de ellos fueron reportados como irritables. En la muestra de la comunidad el $11 \%$ presentaban berrinches, pero $78.4 \%$ fueron reportados como irritables; sin embargo, en la muestra clínica, aunque menos frecuente, la irritabilidad fue asociada a berrinches agresivos. En contraste en la muestra de la comunidad la irritabilidad sólo fue asociada a un pequeño número de niños y niñas con agresividad.

\section{Edad de inicio de los berrinches}

En pocas ocasiones se presenta los berrinches en las etapas iniciales del desarrollo infantil, y es aceptado que se inicien comúnmente a partir de los 18 meses, siendo posible que, en esta edad; al haber alcanzado un buen grado de desarrollo, ya caminen, se comuniquen y experimenten sus emociones con gran intensidad (Koch, 2003). Osterman y Bjorkqvist (2004) encontraron que los berrinches se inician alrededor de los 2 años y su frecuencia disminuye a partir de los 4 años.

\section{Motivos de los Berrinches}

Potegal y Davison (1998) hallaron que las conductas después de los berrinches están relacionadas con la edad, el llanto a gritos, el estrés fisiológico y el inicio de separación de los padres. Manning et al. (2019) los relacionan con un pobre lenguaje expresivo o con disciplina inconsistente, exagerado criticismo, exagerado proteccionismo o negligencia (Fetsch \& Jacobson, 2013).

De acuerdo con la experiencia y los reportes de investigaciones, Eatsman y Rozen (2001) encontraron que las motivaciones que desencadenan berrinches son: presión de la vida cotidiana, conflictos, etapas evolutivas y baja autoestima. 
Presión de la vida cotidiana. Las exigencias de la vida diaria hacen que los padres, madres o cuidadores constantemente estén presionándolos para que se apuren al vestirse, se apuren o terminen de comer, hagan bien las cosas, distingan cuando no es hora de jugar y/o que no pueden tocar ciertos objetos; y el enojo o frustración que provocan estas presiones los desbordan y puede reaccionar con rabietas.

Conflictos. Generalmente en la dinámica familiar hay diversos conflictos, como por ejemplo competencia entre hermanos para que se les preste atención, exigencias de los niños y niñas que los adultos no pueden satisfacer, o exigencias de estos que los niños y niñas no quieran complacer; y aunque los niños tienen distintos niveles de tolerancia, algunos terminan reaccionando con ira y explosiones de cólera.

Etapas evolutivas. Los infantes atraviesan diversas etapas de desarrollo. Las frustraciones son frecuentes cuando en cada fase hay que aprender cosas nuevas y las exigencias del entorno cambian o son mayores. Así, deben aprender a tener autodisciplina y adaptarse a los cambios que involucra el crecimiento.

Baja autoestima. Es importante que en el curso del desarrollo los niños y niñas vayan adquiriendo diversos grados de satisfacción sobre sí mismos y valorar positivamente el cariño o atención que les tengan las personas de su entorno. Es frecuente que a veces los niños se sientan poco atendidos, abandonados y poco queridos; por tanto, no es raro que expresen sus sensaciones de no ser lo suficientemente atendidos con manifestaciones de enojo en forma de berrinches.

Koch (2003) afirma que existen dos puntos de vista sobre los berrinches, uno de ellos plantea que son mecanismos mediante los cuales se descargan y regulan las emociones, es el caso de la explicación basada en el temperamento de los niños y niñas; otro punto de vista hace hincapié en la ansiedad, el manejo de la agresión que surge del inconsciente ya sea por necesidades o frustraciones que nacen por una inmadurez del ego, frecuente dificultad para tomar decisiones y seguir instrucciones, resolución de problemas mediante la agresión física, berrinches, dificultad de separarse de los adultos, no participar con otros niños, llorar y bajar su apetito (California Childcare Health Program, 2006). Se ha dado particular importancia al enojo que experimentan los niños cuando tienen rabietas, ya que surgen generalmente en el hogar con personas con las que suelen sentirse bien. En el enojo de los niños, los padres o cuidadores tiene un rol importante en su disminución, pero cuando los berrinches ocurren en corto periodo de tiempo con incremento de la intensidad, con cambios en el ritmo, cambios en la energía de las vocalizaciones, movimiento y adicionalmente signos fisiológicos como enrojecimiento, no hay dudas de que el niño está muy enojado (Potegal \& Qiu, 2010). 


\section{Curso de los berrinches con el devenir el tiempo}

Cuando los berrinches/rabietas se siguen presentando después de los cuatro años, a pesar de los esfuerzos de los padres y madres y de haber aplicado algunas técnicas de modificación de conducta, mediante el uso de reforzadores positivos y el castigo; es necesario que se derive a un profesional idóneo, cómo el psicólogo o neuropsicólogo, quienes determinarán si es necesario otras evaluaciones y los planes de intervención (Koch, 2003).

Es común aceptar que los niños y niñas demuestren su voluntad y autonomía con conductas que tengan que ver con sus padres o figuras con autoridad, pero su persistencia en intensidad pueden ser indicadores del inicio de un desorden de conducta oposicional desafiante, sobre todo cuando se presenta con excesiva conducta desafiante, falta de remordimiento o sentimientos de culpa por su conducta pasada la crisis, o no hay mayores resultados de la aplicación de técnicas de modificación de conducta cómo el castigo y otros (Baillargeon et al., 2012; Harvey, 2012).

Koch (2003) al investigar berrinches en niños y niñas, encontró que a los 21 meses el $45 \%$ de evaluados del grupo investigado y el $51 \%$ del grupo control manifestaron alguna forma de berrinches y que a medida que aumentaba su edad se disminuía la frecuencia y severidad de ellos. Además, de manera categórica, afirma que las tempranas manifestaciones de berrinches con llanto irritable y reacciones bruscas en los 3 primeros años no son necesariamente predictivas de serios problemas de conducta en el futuro, pero que si los berrinches se prolongan un tiempo, podrían ser predictores de futuros problemas. Según Osterman y Bjorkqvist (2010) los berrinches declinan a partir de los tres años, justo cuando mejoran su lenguaje, que inclusive ayuda a mejorar las conductas agresivas en general.

\section{Diferencias de género y berrinches}

Se cree que los niños suelen ser más activos, desordenados y reactivos que las niñas, pero ambos están en condiciones semejantes para aprender a reaccionar adecuadamente para aliviar la tensión. Lo que está claro es que los adultos les tratan de manera diferente de acuerdo con el sexo del infante. Por lo tanto, no se puede precisar si las diferencias se deben al hecho de ser de uno u otro sexo, o por la manera de que el sexo del infante influye en nuestra forma de tratarlos (Jacklin, 1989). Los estudios que han tomado en cuenta las diferencias de género han sido controversiales, pues los descubrimientos son pocas veces persistentes cuando se replican las investigaciones. Shafer (2000) refiere que las diferencias sólo se perciben en forma clara después de los dos años, pero de todas maneras, hay aceptación de que deben existir diferencias. No es considerado raro que un niño reaccione con un estallido agresivo e inclusive lo podemos pasar por alto, con lo que reforzamos esa conducta y cuando una niña reacciona con llanto fácilmente tratamos de consolarla, reacción que no sucede si es el niño el que llora (Eastman \& Rozen, 2001). 
Koch (2003) encontró que a los 21 meses de edad no se evidenciaron diferencias en los berrinches de niños y niñas, pero a medida que aumenta la edad, la frecuencia y severidad de los berrinches es mayor en niños. A los 9 años la presencia de explosiones de berrinches era por encima del $50 \%$ en los niños, mientras que en las niñas era sólo a un nivel de $30 \%$. Este autor plantea que las respuestas de ira frente a la frustración parecían darse en función de la aceptación cultural. En cuanto a la frecuencia de los berrinches Osterman y Bjorkqvist (2004) no hallaron diferencias entre los niños y niñas de 2 a 4 años.

Heras et al. (2016) hallaron que las niñas poseen mejor nivel emocional global, específicamente en tres de las cuatro dimensiones que la componen: conciencia emocional, regulación emocional y competencia social; solo en habilidades de la vida para el bienestar no obtuvieron superioridad. Por más que en los tiempos modernos vivimos en una sociedad interesada en la igualdad de género, los padres, madres y profesores en la niñez reaccionan de distinta manera; cuando es un niño el que tiene una explosión de enojo que cuando es una niña la que tiene la explosión de ira.

Dado que los hallazgos son contradictorios, el estudio que se presenta pretende saber si en nuestro medio hay diferencias de género en las características de los berrinches. Aquí se plantea que los niños presentan más berrinches que las niñas.

\section{Método}

\section{Diseño}

La investigación es de tipo descriptiva correlacional. Descriptiva porque se aplicó una encuesta para conocer rasgos o conductas de grupo de personas, en este caso, niños y niñas (Kerlinger y Lee, 2002); y correlacional porque se pretende encontrar la relación entre la variable berrinches y la variable género (Salkind, 2012).

\section{Participantes}

De acuerdo con los últimos datos censales disponibles del 2016, fueron matriculados 16,568 menores en edad preescolar en instituciones educativas de Arequipa ( $48.95 \%$ varones y $51.05 \%$ mujeres): 2,570 de 2 años ubicados en cunas, cunas jardín y centros de estimulación temprana (Inicial Ciclo I) y 13,998 de 3 años ubicados en jardines, cunas-jardines y PRONOEI (Inicial Ciclo II). Casi el 80 por ciento de ellos asisten a los 8 distritos más populosos de la provincia: Cerro Colorado, Arequipa, Paucarpata, Cayma, José Luis Bustamante y Rivero, Alto Selva Alegre, Miraflores y Yanahuara. Para la presente investigación los participantes proceden de esos 8 distritos con mayor cantidad de población.

Respetando las proporciones señaladas se logró la participación de las madres de 555 estudiantes matriculados en nivel inicial, el 3.5\% de la población total; de los cuales: $284(51 \%)$ fueron hombres y 271 mujeres (49\%); 183 de dos años, 96 
hombres (52 \%) 87 mujeres $48 \%$ ); 372 de tres años, 188 hombres (51\%) y 184 mujeres (49\%).

\section{Instrumentos}

Se aplicó una Ficha de Reporte de Conductas preparada por los investigadores, donde en su parte inicial incluía datos demográficos. La ficha fue sometida a la revisión de dos psicólogos del medio con amplia experiencia en el tema estudiado. El listado de conductas sobre los berrinches de la Ficha de Reporte está dividido en tres apartados de acuerdo a tres grados de intensidad: leve, moderado y severo. Leve cuando lloray grita, se presentan berrinches una oalgunas veces al mes y dura menos de 5 minutos. Moderado cuando llora y grita, presenta alguna de las conductas de arrojar juguetes/objetos, tirarse al suelo, golpearse a sí mismo con manos y puño, se presenta varias veces a la semana y dura hasta 10 minutos. Severo cuando llora y grita, está presente alguna de las conductas de arrojar juguetes/objetos, tirarse al suelo, golpearse a sí mismo con manos o puños y alguna de las conductas de golpearse contra la pared, el suelo u objetos, agrede físicamente a las personas de su entorno, se presentan diario o varias veces al día y el berrinche dura más de 10 minutos.

\section{Procedimientos}

Se tuvo comunicación con más de una centena de centros de educación inicial que atendían a niños de dos y/o tres años. En las instituciones que hubo respuesta positiva se tuvo una reunión con el personal directivo y docente explicándoles las características de la investigación; resaltando la posibilidad de comunicarse con los padres (madres o cuidadoras principales) ya sea personalmente o por teléfono. Los jardinesy cunas-jardines fueron seleccionados de acuerdo con los siguientes criterios:

- Atendían a niños y niñas de 2 años a 3 años.

- Huboplena colaboración deladirección y personal docente.

- El personal docentetenía buenayperiódica comunicación con los padres y enviaban la agenda de los estudiantes diariamente a los hogares.

- Hubo comunicación por medio de la agenda anunciando que recibirían unas "encuestas" con la motivación y explicación de los objetivos de la investigación.

- Se resaltó la promesa de enviarles una "cartilla de orientación" con orientaciones de cómo manejar problemas de conducta de los infantesy en particular cómo actuar frente a los berrinches.

Fueron excluidas las encuestas en las cuales la edad de los estudiantes era menor a 2 años o mayor de 4 años, igualmente las que no fueron contestadas plenamente y cuando hubo algún reporte de algún tipo de incapacidad del niño o niña.

Inicialmente se aplicó el instrumento a manera de prueba piloto a 23 participantes que no fueron tomados en cuenta en la data final. Ésta sirvió para realizaralgunos reajustes en la aplicación como en la significación 
de algunas palabras de uso en el medio. Además, ayudóa tomar la decisión deubicar los datos de la ficha demográfica en la parte inicial de la encuesta enviada, a fin de que no parezcan como dos instrumentos.

Por medio de las instituciones educativas fueron enviadas un total de 1,325 encuestas a las familias de los niños y niñas. El índice de retorno fue del $42 \%$ es decir 555 fichas de niños y niñas.

Una vez recolectada la data se ha procedido a la calificación y valoración de los instrumentos para lo cual se ha procesado la información medianteel programa del SPSS 24.o, usando la prueba de Chi-cuadrado.

\section{Resultados}

Los resultados se presentan por medio de tablas con frecuencias, porcentajes y con la prueba estadística de Chi Cuadrado cuando fue posible. En primer lugar, considerando las respuestas a las 555 encuestas respondidas, es evidente que la gran mayoría ( $\mathrm{n}=431 ; 77.6 \%)$ de los varones y mujeres de 2 a 3 años presentan reacciones tipificadas como berrinches, solo el $24.4 \%(n=124)$ de los participantes no habían experimentado berrinches. En cuanto a las diferencias de con respecto a la presencia de berrinches entre niños y niñas, no se encontraron diferencias estadísticamente significativas $\left(\mathrm{X}^{2}=0.90\right.$; p> .05), pero 222 niños ( $40 \%$ ) presentaron berrinches al igual que 209 niñas (37.6\%). Por otro lado 62 niños (11.2\%) no presentaron berrinches, de la misma manera 62 niñas (11.2 \%) tampoco presentaron esta conducta.

Con respecto a la relación entre edades de los participantes hombres y mujeres, y la presencia de berrinches, se observa que 144 (33.4\%) tienen 2 años, siendo 75 varones (17.4\%) y 69 mujeres (16.0\%); mientras que de los 287 (66.6 \%) que tienen 3 años, 147 son varones (34.1\%) y 140 mujeres (32.5\%). También se tiene que a los 3 años (66.6\%) hay mayor presencia de berrinches que a los 2 años (33.4\%).

Tabla 1.

Intensidad de los berrinche según el género

\begin{tabular}{|lcccccc|}
\hline & \multicolumn{2}{c}{ Varones } & \multicolumn{2}{c}{ Mujeres } & \multicolumn{2}{c|}{ Total } \\
Intensidad & $\mathbf{f}$ & $\%$ & $\mathrm{~F}$ & $\%$ & $\mathrm{f}$ & $\%$ \\
\hline Leve & 105 & 24.4 & 123 & 28.5 & 228 & 52.9 \\
Moderado & 105 & 24.4 & 80 & 18.6 & 185 & 43.0 \\
Severo & 12 & 2.7 & 6 & 1.4 & 18 & 4.1 \\
Total & 222 & 51,5 & 209 & 48.5 & 431 & 100.0 \\
\hline
\end{tabular}

$\mathrm{X}^{2}=0.40 ; \mathrm{p}<.05$

Se observa en la Tabla 1 que las niñas $\quad 80$ (18.6\%) en nivel moderados y sólo 6 presentan mayor intensidad de berrinches (1.4\%) en nivel severo. En cambio, los con 123 casos $(28.5 \%)$ en un nivel leve, niños presentan mayor intensidad con 12 
casos (2.7\%) en nivel severo y 105 (24.4 son estadísticamente significativas de $\%$ ) en nivel moderado con la misma acuerdo con la prueba Chi cuadrado cantidad en nivel leve. Estas diferencias $\quad\left(\mathrm{X}^{2}=0.40 ; \mathrm{p}<.05\right)$.

Tabla 2.

Características de los berrinches según el género

\begin{tabular}{|c|c|c|c|c|}
\hline \multirow{3}{*}{ Características de los berrinches } & \multicolumn{4}{|c|}{ Género } \\
\hline & \multicolumn{2}{|c|}{ Varones } & \multicolumn{2}{|c|}{ Mujeres } \\
\hline & f & $\%$ & f & $\%$ \\
\hline \multicolumn{5}{|l|}{ Llora } \\
\hline Sí & 203 & 91.4 & 197 & $94 \cdot 3$ \\
\hline No & 19 & 8.6 & 12 & $5 \cdot 7$ \\
\hline Total & 222 & 100.0 & 209 & 100.0 \\
\hline \multicolumn{5}{|l|}{ Grita } \\
\hline Sí & 175 & 78.8 & 165 & 78.9 \\
\hline No & 47 & 21.2 & 44 & 21.1 \\
\hline Total & 222 & 100.0 & 209 & 100.0 \\
\hline \multicolumn{5}{|l|}{ Arroja juguetes } \\
\hline Sí & 128 & $57 \cdot 7$ & 92 & 44.0 \\
\hline No & 94 & 42.3 & 117 & 56.0 \\
\hline Total & 222 & 100.0 & 209 & 100.0 \\
\hline \multicolumn{5}{|l|}{ Se tira al suelo } \\
\hline Sí & 92 & 41.4 & 74 & $35 \cdot 4$ \\
\hline No & 130 & 58.6 & 135 & 64.6 \\
\hline Total & 222 & 100.0 & 209 & 100.0 \\
\hline \multicolumn{5}{|l|}{ Se golpea a sí mismo } \\
\hline Sí & 15 & 6.8 & 5 & 2.4 \\
\hline No & 207 & 93.2 & 204 & 97.6 \\
\hline Total & 222 & 100.0 & 209 & 100.0 \\
\hline \multicolumn{5}{|l|}{ Se golpea contra la pared } \\
\hline Sí & 5 & 2.3 & $\mathbf{1}$ & 0.5 \\
\hline No & 217 & $97 \cdot 7$ & 208 & $99 \cdot 5$ \\
\hline Total & 222 & 100.0 & 209 & 100.0 \\
\hline \multicolumn{5}{|l|}{ Agrede físicamente } \\
\hline Sí & 73 & 32.9 & 51 & $24 \cdot 4$ \\
\hline No & 149 & 93.2 & 158 & 75.6 \\
\hline Total & 222 & 100.0 & 209 & 100.o \\
\hline
\end{tabular}


La Tabla 2 muestra que de las 431 familias que respondieron en la ficha de reporte las características de berrinches en niños y niñas de 2 y 3 años, se distinguen siete características básicas: 203 niños (91.4\%) y 197 niñas (94.3\%) lloran, 175 niños (78.8 $\%)$ y 165 niñas (78.9 \%) gritan, 128 niños
(57.7\%) y 92 niñas ( $44.0 \%$ ) arrojan juguetes, 92 niños (41.4\%) y 74 niñas (35.4\%) se tiran al suelo, 15 niños $(6.8 \%)$ y 5 niñas (2.4\%) se golpean a sí mismos, 5 niños (2.3\%) y 1 niña (0.5\%) se golpean contra la pared, y finalmente, 73 niños (32.9\%) y 51 niñas (24.4\%) agreden físicamente.

Tabla 3.

Frecuencia de berrinches y género

\begin{tabular}{|lcccc|}
\hline & \multicolumn{3}{c}{ Género } \\
& \multicolumn{2}{c}{ Varones } & \multicolumn{2}{c|}{ Mujeres } \\
& f & \% & f & $\%$ \\
\hline Algunas veces al mes & 106 & 47.7 & 102 & 48.8 \\
Varias veces/semana & 90 & 40.5 & 80 & 38.3 \\
Diario/varias al día & 26 & 11.7 & 27 & 12.9 \\
Total & 222 & 100.0 & 209 & 100.0 \\
\hline
\end{tabular}

$\mathrm{X}^{2}=0.864 ; \mathrm{p}>.05$

La frecuencia de berrinches en niños y niñas son descritas en la Tabla 3, comprendida por la muestra que de 431 familias que respondieron la ficha de reporte, en la cual 106 niños (47.7\%) y 102 niñas ( 48.8 $\%)$ presentan berrinches algunas veces al mes; 90 niños (40.5 \%) y 80 niñas (38.3 $\%)$ presentan berrinches varias veces a la semana, 26 niños (11.7 \%) y 27 niñas (12.9\%) presentan berrinches diaria o varias veces al día. Sin embargo, no se encontraron asociaciones estadísticamente significativas entre la frecuencia y el género de los niños mediante la prueba Chi cuadrado $\left(\mathrm{X}^{2}=0.864 ; \mathrm{p}>.05\right)$.

Tabla 4.

Duración de berrinches y género

\begin{tabular}{|lcccc|}
\hline \multirow{2}{*}{ Duración } & \multicolumn{4}{c}{ Género } \\
& Farones & \% & f & \%ujeres \\
\hline Menos de 5 minutos & 165 & 74.7 & 159 & 76.1 \\
Hasta 1o minutos & 43 & 19.5 & 41 & 19.6 \\
Más de 1o minutos & 13 & 5.9 & 9 & 4.3 \\
Total & $\mathbf{2 2 2}$ & 100.0 & 209 & 100.0 \\
\hline
\end{tabular}

$\mathrm{X}^{2}=0.652 ; \mathrm{p}>.05$ 
La Tabla 4 muestra la duración de berrinches en niños y niñas de 2 y 3 años. Se describen tres categorías: 165 niños $(74.7$ \%) y 159 niñas (76.1\%) tienen una duración de berrinches menor de 5 minutos, 43 niños $(19.5 \%)$ y 41 niñas $(19.6 \%)$ tienen una duración de hasta 10 minutos, $\mathrm{p}>.05)$.

Tabla 5.

¿Cuándo se Presentan los Berrinches?

\begin{tabular}{|lcc|}
\hline & f & $\%$ \\
\hline No le dan el objeto & & \\
Sí & 315 & 73.9 \\
No & 111 & 26.1 \\
Total & 426 & 100.0 \\
\hline No se realiza la acción & & \\
Sí & 243 & 57.0 \\
No & 183 & 43.0 \\
Total & 426 & 100.0 \\
\hline No se le entiende & & \\
Sí & 139 & 32.6 \\
No & 287 & 67.4 \\
Total & 426 & 100.0 \\
\hline
\end{tabular}

La Tabla 5 describe cuándo se presentan años manifiestan berrinches cuando los berrinches en niños y niñas de 2 y 3 no le dan el objetivo que pide, el 57 años. Respondieron 426 familias este \% cuando no se realiza la acción que punto en la ficha de reportes. En ella quiere, mientras que el $32.6 \%$ tiene se identifican 3 situaciones básicas: berrinches cuando no se le entiende el $73.9 \%$ de los niños y niñas de 2 y 3 lo que desea o quiere. 
Tabla 6.

¿Cómo se le Pasan los Berrinches?

\begin{tabular}{|lcc|}
\hline & f & $\%$ \\
\hline Se le da el objeto que pide & & \\
Sí & 161 & 37.7 \\
No & 266 & 62.3 \\
Total & 427 & 100.0 \\
\hline Se realiza la acción que quiere & & \\
Sí & 97 & 22.7 \\
No & 330 & $77 \cdot 3$ \\
Total & 427 & 100.0 \\
\hline Lo consuelan y cargan en brazos & & \\
Sí & 356 & 83.4 \\
No & 71 & 16.6 \\
Total & 427 & 100.0 \\
\hline Se aleja y el niño(a) lo sigue & & \\
Sí & 190 & 44.5 \\
No & 237 & 55.5 \\
Total & 427 & 100.0 \\
\hline Se aleja y no permite que el niño(a) lo siga & & \\
Sí & 66 & 15.3 \\
No & 361 & 84.7 \\
Total & 427 & 100.0 \\
\hline Viene otra persona, lo atiende y se calma & & \\
Sí & 298 & 69.9 \\
No & 427 & 100.0 \\
Total & & \\
\hline
\end{tabular}

En la Tabla 6 se describe cómo se les pasan los berrinches a los niños y niñas de 2 y 3 años. Respondieron a esta pregunta 427 familias y en la ficha de reporte se han identificado 6 situaciones básicas: el $37.7 \%$ de personas a cargo de ellos le dan el objeto que pide, lo atiende y se calma. el $22.7 \%$ realiza la acción que quiere, el $83.4 \%$ le habla, lo calma y carga en brazos, el $44.5 \%$ se aleja y el niño o niña lo sigue, el $15.3 \%$ se aleja y no permite que el niño(a) lo siga, mientras que el $30.1 \%$ refiere que viene otra persona, 
Tabla 7 .

Lugar dónde se presentan los berrinches según el género

\begin{tabular}{|c|c|c|c|c|c|c|}
\hline \multirow{4}{*}{$\begin{array}{l}\text { Dónde se presentan } \\
\text { Casa }\end{array}$} & \multicolumn{4}{|c|}{ Género } & \multirow{2}{*}{\multicolumn{2}{|c|}{ Total }} \\
\hline & \multirow{2}{*}{\multicolumn{2}{|c|}{$\begin{array}{c}\text { Varones } \\
\text { f \% }\end{array}$}} & \multirow{2}{*}{\multicolumn{2}{|c|}{$\begin{array}{c}\text { Mujeres } \\
\text { f \% }\end{array}$}} & & \\
\hline & & & & & \multirow{2}{*}{$\frac{f}{214}$} & \multirow{2}{*}{$\frac{\%}{52.4}$} \\
\hline & 107 & 26.2 & 107 & 26.2 & & \\
\hline Lugares públicos & 14 & 3.4 & 18 & 4.4 & 32 & 7.8 \\
\hline Centro educativo & 7 & 1.7 & o & 0.0 & 7 & 1.7 \\
\hline Casa y lugares públicos & 64 & 15.6 & 58 & 14.2 & 122 & 29.8 \\
\hline Casa y centro educativo & 6 & 1.5 & 4 & 1.0 & 10 & 2.5 \\
\hline Todos los lugares & 18 & $4 \cdot 4$ & 6 & 1.4 & 24 & 5.8 \\
\hline Total & 216 & 52.8 & 193 & 47.2 & 409 & 100.0 \\
\hline
\end{tabular}

En la Tabla 7 se tienen en respuestas de las 409 familias que respondieron a la pregunta con respecto al lugar donde se presentan los berrinches según el género. Se tiene que 107 niños $(26.2 \%)$ y 107 niñas $(26.2 \%)$ presentan berrinches en casa, 14 niños (3.4\%) y 18 niñas (4.4\%) las tienen en lugares públicos,
7 niños ( $1.7 \%$ ) y ninguna niña en las tienen en el centro educativo, 64 niños (15.6\%) y 58 niñas (14.2\%) en casa y en lugares públicos, 6 niños (1.5\%) y 4 niñas (1\%) en casa y el centro educativo, mientras que 18 niños (4.4\%) y 6 niñas (1.4\%) presentaron berrinches en todos los lugares.

Tabla 8.

\section{Intensidad de los berrinches y ser hijo único}

\begin{tabular}{|lcccccc|}
\hline $\begin{array}{l}\text { Intensidad } \\
\text { berrinche }\end{array}$ & \multicolumn{2}{c}{$\begin{array}{c}\text { Sí Hijo único } \\
\text { f \% }\end{array}$} & \multicolumn{2}{c}{$\begin{array}{l}\text { No } \\
\text { f \% }\end{array}$} & F & Total \\
\hline Leve & 103 & 24.6 & 119 & 28.5 & 222 & 53.1 \\
Moderada & 91 & 21.8 & 89 & 21.3 & 170 & 43.1 \\
Severa & 12 & 2.9 & 4 & 0.9 & 16 & 3.8 \\
Total & 206 & 49.3 & 212 & 50.7 & 418 & 100.0 \\
\hline
\end{tabular}

Cuatrocientos dieciocho familias respondieron en la ficha de reporte para procesar los datos con respecto a la intensidad de los berrinches y la condición de ser hijo único o no. En la Tabla 8 se observa que 103 niños y niñas hijos únicos (24.6\%) y 119 no hijos únicos (28.5\%) presentan berrinches leves, 91 hijos únicos $(21.8 \%)$ y 89 no hijos únicos (21.3\%) presentan berrinches moderados, y finalmente, 12 hijos únicos $(2.9 \%)$ y 4 no hijos únicos (o.9\%) presentan berrinches severos. 
Tabla 9.

Tipo de familia e incidencia de berrinches

\begin{tabular}{|lcccccc|}
\hline \multirow{1}{*}{ Tipo de familia } & \multicolumn{3}{c}{ Sí } & \multicolumn{9}{c}{ Berrinches } \\
& f & $\%$ & f & $\%$ & fo & $\%$ \\
\hline Nuclear & 213 & 51.1 & 60 & 57.1 & 273 & 52.3 \\
Monoparental & 44 & 10.6 & 8 & 7.6 & 52 & 10.0 \\
Extensa & 142 & 34.0 & 32 & 30.5 & 174 & 33.3 \\
Reconstituida & 18 & 4.3 & 5 & 4.8 & 23 & 4.4 \\
Total & 417 & 100.0 & 105 & 100.0 & 522 & 100.0 \\
\hline
\end{tabular}

$\mathrm{X}^{2}=0.643 ; \mathrm{p}>.05$

La Tabla 9 muestra las respuestas de las 522 familias que respondieron la ficha sociodemográfica con los cuatro tipos de familia y su cruce con la intensidad de los berrinches, donde se aprecia que 213 de niños y niñas $(51.1 \%)$ con presencia de berrinches y $60(57.1 \%)$ sin presencia de berrinches pertenecen a la familia nuclear, $44(10.6 \%)$ con presencia de berrinches y $8(7.6 \%)$ sin presencia de berrinches pertenecen a la familia monoparental, 142 (34\%) con presencia de berrinches y 32 (30.5\%) sin presencia de berrinches pertenecen a la familia extensa, y finalmente, $18(4.3 \%)$ con presencia de berrinches y $5(4.8 \%)$ sin presencia de berrinches pertenecen a la familia reconstituida. Sin embargo, la asociación entre el tipo de familia y la incidencia de berrinches no es estadísticamente significativa tras aplicar la prueba Chi cuadrado $\left(\mathrm{X}^{2}=0.643 ; \mathrm{p}>.05\right)$.

\section{Discusión}

La hipótesis planteada de que los varones de 2 años a 3 años presentarían mayor incidencia de berrinches en comparación con las mujeres de la misma edad, no ha sido confirmada. Si bien estos resultados no han sido los esperados, ya Shafer (200o), refería que los resultados muestran diferencias de temperamento entre niños y niñas, pero estas diferencias no son claras y no se asocian de manera evidente con las condiciones biológicas. Los resultados hallados en la presente investigación pueden deberse a que en los tiempos actuales, se promueve más la igualdad de género, y los estilos de crianza moderna han ido variando con el paso del tiempo. Por tanto, es posible que los padres, madres, docentes y otros adultos reaccionen de semejante manera cuando un niño o niña tienen explosiones de enojo y no como en el pasado en que se aceptaba más estas conductas en los niños y en las niñas no, ya que en las niñas no era femenino reaccionar de esa manera (Jacklin, 1989; Eastman \& Rozen, 2001). Es decir, que ahora, a la luz de nuestros resultados, tanto niños como niñas pueden reaccionar con berrinches para descargar su tensión. Es posible entonces, que la mayoría de padres modernos estén tratando de manera similar a sus hijos e hijas ya sean hombres o mujeres. 
Otra explicación, más de tipo cultural, es que biológicamente tanto los niños como las niñas tienen maneras similares de expresar sus emociones, pero recién en los años posteriores se hace evidente la influencia de la sociedad en las reacciones consideradas como masculinas o femeninas, y a partir de ello se hacen claras las influencias de la manera en cómo los adultos los tratan, con lo cual se hace referencia al rol de género. Si bien Shaffer (2000) afirmaba que el grado de conformidad con las expectativas sociales es mayor a medida que las personas aumentan de edad, las niñas tienden a conformarse mejor a diferencia de los niños, por lo tanto, son ellos quienes suelen presentar más berrinches o rabietas.

Los resultados de la investigación concuerdan con los hallazgos de Koch (2003) quien no encontró diferencias en los berrinches de varones y mujeres a los 21 meses de edad, aunque también encontró que al aumentar la edad (a los 9 años), aumentan la frecuencia y severidad de los berrinches en los varones. Igualmente Osterman y Bjorkqvist (2004), no hallaron diferencias en los berrinches entre niños y niñas de 2 a 4 años. En relación con área emocional Heras et al. (2016) señalaron que, si bien las niñas poseen mejor desarrollo emocional, los padres y profesores en la niñez reaccionan de distinta manera cuando se trata de un niño o una niña cuando tiene una explosión de enojo o de ira.

Es necesario señalar que, en las 555 fichas de registro de conductas, se halla que la gran mayoría $431(77.6 \%)$ presentan berrinches, resultados que confirman la aceptación de que los berrinches son manifestaciones consideradas frecuentes y normales en la infancia. $\mathrm{Al}$ respecto, Green et al. (2011) señalaron la posibilidad de que los berrinches sirvan para expresar emociones fuertes que vivencian los niños, como la ira o tristeza, y que paulatinamente aprendan a manejarlas. Igualmente, Eastman y Rozen (2001), afirman que los berrinches son formas de expresar las vivencias negativas que experimentan los niños y niñas. Asimismo, Wakschlaq et al. (2012) resaltan que el $83.7 \%$ de niños en etapa preescolar presentaron berrinches alguna vez, estos hallazgos guardan semejanza con los resultados encontrados en esta investigación. Es de notar que Novotney (2012), encontró que sólo el $10 \%$ de niños en la etapa preescolar presentan berrinches, esto en contraste con los resultados de esta investigación en la cual se observa que el $77.6 \%$ presentan berrinches.

En cuanto a edad, la American Academy of Pediatrics (1989) señala que las explosiones de ira y los berrinches se presentan en todas las edades de la infancia, pero son más frecuentes entre los 18 meses hasta los tres años. Belden et al. (2008) afirman que el $70 \%$ presentan berrinches entre los 18 y 60 meses. Koch (2003) refirió por otro lado, que a medida que aumentan de edad la severidad de los berrinches es mayor. En la presente investigación es muy claro, de las 431 familias que confirmaron la presencia de berrinches, que estos son significativamente más frecuentes a los 3 años que a los 2 años, indistintamente del género de los participantes. 
Si bien no se demostró el que los niños tengan mayor incidencia de berrinches, se halló que los niños de 2 a 3 años presentaban berrinches con mayor intensidad que en las niñas de 2 a 3 años. Estas diferencias de mayor intensidad de los berrinches en los varones son estadísticamente significativas. Parece ser que los niños son más fáciles de frustrarse y reaccionan más fácilmente con ira que las niñas. Al respecto, Koch (2003) refirió que a los 21 meses de edad no se encontraban manifiestas diferencias entre los berrinches de niños y niñas, pero que a medida que aumentan de edad la severidad de los berrinches es mayor en los varones. Wakschlag et al. (2012) han encontrado que los berrinches diarios no son tan típicos y Carlson et al. (2016), al estudiar muestras clínicas y de la comunidad, hallaron que los berrinches en muestras clínicas son más intensos que en las segundas. Asimismo, Eastman y Rozen (2001) afirman que los berrinches pueden considerarse como un problema serio cuando son muy intensos.

El llanto, gritos, arrojar juguetes y tirarse al suelo son las características más frecuentes en los berrinches estudiados. En el pasado, Kanner (1935) refería que los berrinches se caracterizan principalmente por inicio rápido o gradual de llanto a gritos y zapateo, expresiones de insultos en voz alta y en casos más serios, patear el suelo, agitar sus brazos, empujar o golpear a las personas cercanas, arrojar objetos, tirarse al suelo, golpearse a sí mismo la cabeza contra las paredes u otro objeto. De manera similar, de acuerdo con la American Academy of Pediatrics
(1989) se acepta que el llanto, gritos, pataletas y hasta agresiones son las características más frecuentes. Potegal y Davidson (2003) indicaron también que las conductas de agredirse a sí mismo o a los demás son más frecuentes en los niños que en las niñas.

Eastman y Rozen (2001) consideran que los berrinches pueden considerarse un problema cuando son demasiado frecuentes (varias veces a la semana). Potegal et al. (2003) aseveran que la frecuencia de los berrinches es de uno por día, pero, Wakschlag et al. (2012) reportaron que los berrinches diarios no son tan típicos y sólo los presentan el $10 \%$ de los preescolares. En la muestra estudiada, los berrinches se presentan en un $48.8 \%$ algunas veces al mes, $38.3 \%$ varias veces a la semana y 12.9 $\%$ diariamente; que de alguna forma son similares a las cantidades reportadas en estudios previos. En cuanto a la relación frecuencia y género no se han hallado diferencias significativas, los porcentajes son similares en la frecuencia mensual (varones $47.7 \%$ y mujeres $48.8 \%$ ), semanal (varones 40.5 $\%$ y mujeres $38.3 \%$ ); y diaria (varones $11.7 \%$ y mujeres $12.9 \%)$.

Con respecto a la duración de berrinches, en la gran mayoría de varones $(74.7 \%)$ como de las mujeres $(76.1 \%)$ se encontró un lapso de menos de 5 minutos. En menor frecuencia los berrinches son hasta de 10 minutos, y la duración de los berrinches es mayor de 10 minutos en bajos porcentajes (varones 5.9 \% y mujeres $4.3 \%$ ). Tampoco se halló 
relación entre el género y la duración de los berrinches. Nuestros resultados son cercanos a lo encontrado por Osterman y Bjorkqvist (2004), quienes en su informe refieren que el tiempo de duración en los infantes finlandeses fue entre los 5 y 10 minutos, pero que berrinches de más de media hora se presentaron en $6 \%$ de los casos. Para estos investigadores los berrinches que duran más de 10 minutos deben ser evaluados y seguidos ya que en el futuro pueden continuar y dejar de ser un berrinche para convertirse en un problema de conducta.

En el presente trabajo los berrinches se presentan cuando a los niños y niñas no se les da lo que piden ( $73.9 \%)$, no se realiza la acción que quieren ( $57 \%$ ) y no se le entiende en lo que desean o quieren (32.6\%). Todas esas situaciones les causan frustración, de forma similar a lo mencionado por la American Academy of Pediatrics (1989). Es posible que estas frustraciones estén mezcladas con tristeza o quejas que buscan llamar la atención sobre lo que se desea (Green et al., 2011). Al respecto, Kanner (1935) afirmaba que los berrinches deberían ser considerados como malos hábitos.

En el presente estudio se aprecia que en la frecuencia de berrinches de algunas veces al mes los porcentajes son similares en niños y niñas, con $47.7 \%$ y $48.8 \%$, respectivamente. La frecuencia acumulada de berrinches diarios o varias veces al día y varias veces a la semana es alta, por tanto a pesar de que se considere que los berrinches son expresiones normales en la niñez, su presencia afecta la vida del hogar. Asimismo, Osterman y Bjorkqvist (2004) no encontraron diferencias significativas en la frecuencia de berrinches, sin embargo reportan que el $37.3 \%$ presentan berrinches una vez a la semana, mientras que en la presente investigación se encontró que el promedio reportado en la frecuencia de berrinches es en el 48.3 $\%$, de una vez al mes.

Generalmente las estrategias que usan los adultos para que se les pasen los berrinches a los niños, son proporcionarles lo deseado en el $37.7 \%$ de los casos, hacer la acción que solicitan en el $22.7 \%$; aunque la gran mayoría, es decir, un $83.4 \%$, además de darle o ejecutar lo deseado, tratan de calmarlo hablándole con dulzura y cargándolo, pero algunos adultos recurren a alejarse del niño o niña. Es posible que los padres y madres ante estas situaciones sientan la necesidad de calmar los berrinches en el menor tiempo posible con estas acciones, acompañado con cierto grado de sensibilidad y empatía, la reacción de satisfacer lo deseado para que se cesen rápidamente los berrinches; pero también con ello es posible que se les refuerce tales conductas por lo tanto los berrinches pueden persistir a lo largo del tiempo. Si bien Geleerd (1944) recomendaba dejarlo solo hasta que se calme o ser cambiado de lugar si destruía cosas, en general sugería no tratarlos con firmeza ante los berrinches, quizá porque los berrinches eran considerados como expresiones normales en esa etapa del desarrollo. Al respecto, Osterman y Bjorkqvist (2010) afirman 
que los berrinches son más fáciles de controlar cuando mejoran su lenguaje.

En las 409 familias que respondieron a la pregunta de dónde se presentan los berrinches, se halló que mayormente los berrinches son manifestados en casa 52.4 $\%$, seguido de en casa y lugares públicos

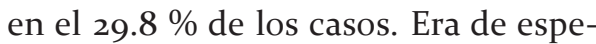
rarse que la mayoría de los berrinches se presentan en el hogar donde, ya que allí pueden manifestar con mayor libertad sus emociones, por ello cuando se trata de manejar los berrinches es necesario la participación de todos los miembros que habitan en el hogar. Es probable que la significación del berrinche sea mayor cuando se presentan en dos o más lugares, generalmente el hogar y en lugares públicos, sobre todo las tiendas ya que los niños suelen desear que les compren cosas que los adultos inicialmente se niegan a comprarles, pero producido el berrinche logran que les den lo que piden. Estos hallazgos son semejantes a las investigaciones de Osterman y Bjorkqvist (2004) quienes afirman que los berrinches en niños y niñas se presentan con mayor frecuencia en la casa y dentro de los vehículos.

En la muestra estudiada menos de la mitad son hijos únicos $49.3 \%$, es probable que esta alta proporción se deba a que las familias estudiadas son jóvenes. Es de notar que el berrinche de tipo leve es proporcionalmente similar en hijos únicos y no únicos, aunque si bien los porcentajes son bajos, $2.9 \%$ y $0.9 \%$ respectivamente, el porcentaje de berrinches severos en hijos únicos es el triple de los no únicos. Se acepta que como los hijos únicos tienen más probabilidad de que sean satisfechos todos sus deseos, se frustren fácilmente cuando se les niegue algo y hayan aprendido a tener berrinches más intensos con el fin de lograr lo que quieren o desean. Lamentablemente, en este punto, no se encontraron investigaciones que contrasten con los hallazgos obtenidos.

No se encontró una relación estadísticamente significativa entre el tipo de familia y la presencia de berrinches, sin embargo para Eastman y Rozen (2001), la presencia de berrinches se debe a la forma de reaccionar de los padres y madres, ya que ésta puede variar si se trata de un sólo progenitor o si están presentes ambos. En un caso es una sola persona la que debe tomar decisiones sobre sus reacciones, en el otro caso, se debe tomar en cuenta el estilo del otro progenitor, lo que puede resultar en apoyo o discrepancia en este tipo de situaciones. Por otro lado, se puede observar que los berrinches predominan en el tipo de familia nuclear, donde se obtuvieron los porcentajes más elevados, posiblemente, a que la discrepancia entre los padres contribuye a la expresión de este tipo de conductas.

En cuanto a las limitaciones del estudio, la más importante fue lograr la participación de los padres, que si bien el deseo fue hacerlo presencialmente, solo se pudo conseguir mediante el envío de los instrumentos a través de las profesoras. A pesar de ello, y de 
tratarse de una muestra comunitaria, puede concluirse que la incidencia de berrinches en los niños y niñas es alta. Aunque en frecuencia no hubo diferencias de género, si se halló que, significativamente, los varones presen$\tan$ mayor intensidad en los berrinches que las niñas.

\section{Financiamiento}

La investigación fue autofinanciada.

\section{Conflictos de interés}

Los autores declaran que no tienen conflictos de interés. 


\section{Referencias}

American Academy of Pediatrics (1989). Los berrinches y rabietas: Un elemento normal del crecimiento. Medem. http://www.mipediatra.com/pdf/berrinches-print.pdf

Ashdown, D., \& Bernard, M. (2011). Can explicit instruction in social and emotional learning skills benefit the social-emotional development, wellbeing, and academic achievement of young children? Early Childhood Educational Journal, 39(6), 397-405.

Askeland, D. (2019). Social emotional development in early childhood. (Tesis de grado) Northwestern College, USA.

Baillargeon, R. H., Keenan, K, \& Cao, G. (2012). Oppositional and defiant behaviors in toddlers: Maybe those aren't just temper tantrums. Journal of Developmental $\mathcal{E}$ Behavioral Pediatrics, 33, 6o8-617.

Bee, H. (1992). The developing child. Harper Collins.

Belden, A. C., Thomson, N. R., y Luby, J. L. (2008). Temper Tantrums behaviors as marker of clinical problems. Clinician's Research Digest. Briefings in Behavioral Science, 26(7). www.apa.org/journlas/crd

California Childcare Program (2006). Desarrollo social y emocional de los niños. Department of Education Child Development Division y de Federal Maternal and Child Health Bureau. https://cchp.ucsf.edu/sites/cchp.ucsf.edu/files/15_ CCHA_SP_SocialEmot_o6o6_v3.pdf

Carlson, G. A., Danzig, A. P., Dougherty, L. R., Bufferd, S. J., \& Klein, D. N. (2016). Loss of temper and irritability: The relationship to tantrums in a continuity and clinical sample. Journal of Child and Adolescent Psychopharmacology, 26, 114-122.

Daniels, E., Mandleco, B., \& Luthy, K. (2012). Assessment, management, and prevention of childhood temper tantrums. Journal of the American Academy of Nurse Practitioners, 24, 569-573.

Darling-Churchil, K. E., \& Lippman, L. (2016). Early childhood social and emotional development: advancing the field of measurement. Journal of Applied Developmental Psychology, 45, 1-7. 
Davison, D. H. (2003). Temper tantrums in young children. Children and Family. University of Hawaii at Manoa.

Eastman, M., \& Rozen, S. C. (2001). No más berrinches. Consejos para lograr armonía entre padres e hijos. Aguilar.

Fetsch, R. J., \& Jacobson, B. (2013). Children s anger and tantrums. Consumer Series Family. Extension. Fact Sheet. Colorado State University.

Geleerd . L. R. (1944). Observations on temper tantrums in children. Kansas Meeting.

Green, J. A., Whitney, P. G., \& Potegal, M. (2011). Screaming, yelling, whining, and crying: Categorical and intensity differences in vocal expressions of anger and Children's tantrums. American Psychological Association, 11, 1124-1133.

Harvey, K. (2012). Preventing abusive head trauma: Two promising new strategies to and toddler tantrums. Workshop, Session 128.

Heras, D., Copa, A., \& Lara, F. (2016). Desarrollo emocional en la infancia, un estudio sobre las competencias emocionales de niños y niñas. Revista de Psicología, 1(1), 67-74.

Jacklin, C. N. (1989). Female and male: Issues of gender. American Psychologist, $44,127-133$.

Kanner, L. (1935). Emotional disorders. En L. Kanner (Ed.), Child psychiatry (pp, 275-293). Charles C. Thomas.

Kerlinger, F., \& Lee, H. (2002). Investigación del comportamiento. McGraw-Hill.

Koch, E. (2003). Reflections on a study of temper tantrums in older children. Psychoanalytic Psychology, 20, 456-471.

Manning, B. L., Roberts, M. Y., Estabrook, R., Petitclerc, A., Burns, J., Briggs-Gowan, M., Wakschlag, L. S., \& Norton, E. (2019). Relations between toddler expressive language and temper tantrums in a community sample. Journal of Applied Developmental Psychology, 65, 1-10. https://doi.org/10.1016/j.appdev.2019.101070

Mireault, G., \& Trahan, J. (2007). Tantrums and anxiety in early childhood: a pilot study. Childhood Research Practice, 9(2), 1-10. https://ecrp.uiuc.edu/v9nz/ mireault.html 
Novotney, A. (2012). Daily temper tantrums aren't normal for preschoolers. Monitor on Psychology, 43, 10.

Osterman, K., \& Bjorkqvist, K. (2004). Temper tantrums among young children. Conference Society for Research on Aggression. XVI World Meeting. USA.

Osterman, K., \& Bjorkqvist, K. (2010). A cross-sectional study of onset, cessation, frequency, and duration of children's temper tantrums on a nonclinical sample. Psychological Reports, 106, 448-454.

Papalia, D. E., Olds, S. W., \& Feldman, R. D: (2001). Psicología del desarrollo. McGraw-Hill.

Potegal, M., \& Davidson, R. J. (1998). Young children s post tantum affiliation with their parents. Aggressive Behavior, 23(5).

Potegal, M., \& Davidson, R. J. (2003). Temper tantrums in young children: behavioral composition. Journal of Developmental \& Behavioral Pediatrics, 24, 140-147. http://journals.lww.com/jrnldbp/abstract//2003/o6ooo/ Temper-Tantrums-in-Young-Children-1-Behavioral.2.aspx/

Potegal, M., \& Qiu, P. (2010). Anger in children's tantrums: A new, quantitative, behaviorally based model. En M. Potegal, G. Stemmler, \& C. Spielberger (Eds.), International Handbook of Anger: Constituent and concomitant biological, psychological, and social processes (pp. 193-217). Springer. https://doi. org/10.1007/978-0-387-89676-2-12

Potegal, M., Kosorok, M. R., \& Davidson, R. J. (2003). Temper tantrums in young children: tantrum duration and temporal organization. Journal of Developmental E Behavioral Pediatrics, 24, 148-154.

Rice, F. P. (1997). Desarrollo del ciclo vital. Estudio del ciclo vital. Prentice-Hall.

Romano, E., Babchishin, L., Pagani, L. S., \& Kohen, D. (2010). School readiness and later achievement: Replication and extension using a nationwide Canadian survey. Developmental Psychology, 46(5), 995-1007. https://doi.org/10.1037/aoo18880

Salkind, N. J. (2012). Exploring research. 8va ed. Pearson.

Shaffer, D. R. (200o). Psicología del desarrollo. Infancia y adolescencia. Thomson. 
Solter, A. (2020). Understanding tears and tantrums, Aware Parenting Institute. www.awareparenting.com

Thomas, A., Chess, S., \& Birch, H. G. (1970). The origin of personality. Scientific American, 223(2), 102-109. https://doi.org/10.1038/scientificamericano870-102

Thompson, R. A. (2018). Social-emotional development in the first three years. Establishing the foundation. The Pennsylvania State University.

Tortosa, F. \& Mayor, L. (1992). Watson y la psicología de las emociones: Evolución de una idea. Psicothema, 4, 297-315.

Wakschlag, L. S., Choi, S. W., Carter, A. S., Hullsiek, H., Burns, J., McCarthy, K. Leibenluft, E., \& Briggs-Gowan, M. J. (2013). When Temper Tantrums shift from normal to problematic. Clinician's Research Digest, 53, 1099-1108. https://doi. org/10.111/j.1469.7610.2012.02595.X

Recibido: 11 de octubre de 2020

Revisado: 6 de noviembre de 2020

Aceptado: 18 de junio de 2021 